\title{
Using highly excited baryons to catch the quark mass
}

\author{
T. Van Cauteren ${ }^{1, a}$, P. Bicudo ${ }^{2}$, M. Cardoso ${ }^{2}$, and Felipe J. Llanes Estrada ${ }^{3}$ \\ 1 Dept. Physics and Astronomy, Ghent University, Proeftuinstraat 86, B-9000 Gent, Belgium \\ 2 Dpto. Física, Instituto Superior Técnico, Avda. Rovisco Pais, 1096 Lisbon, Portugal \\ 3 Dpto. Física Teórica I, Universidad Complutense, Avda. Complutense s/n, 28040 Madrid, Spain
}

\begin{abstract}
Chiral symmetry in QCD can be simultaneously in Wigner and Goldstone modes, depending on the part of the spectrum examined. The transition regime between both, exploiting for example the onset of parity doubling in the high baryon spectrum, can be used to probe the running quark mass in the mid-IR power-law regime. In passing we also argue that three-quark states naturally group into same-flavor quartets, split into two parity doublets, all splittings decreasing high in the spectrum. We propose that a measurement of masses of high-partial wave $\Delta^{*}$ resonances should be sufficient to unambiguously establish the approximate degeneracy and see the quark mass running. We test these concepts with the first computation of the spectrum of high-J excited baryons in a chiral-invariant quark model.
\end{abstract}

\section{Introduction}

In the last thirty years, Quantum Chromodynamics has turned out to give an accurate description of many high-energy processes. In the last decennium, a lot of effort has gone to solving the QCD equations on a discretized spacetime lattice, using an impressive amount of computing power. This, together with perturbation theory, has led to very persuasive evidence that QCD indeed describes the strong interaction, both at the high-energy end and at the static end. However, there still remains the question if the theory describes the transition between the perturbative and non-perturbative domain. A suitable quantity for checking this is the quark mass, which should be a function of the quark's momentum due to the interactions with the QCD medium. Asymptotic freedom tells us that at high quark momentum $k$, the nonstrange quark mass should approach the current quark mass of the order of $1-5 \mathrm{MeV}$ as deduced from chiral perturbation theory or sum-rules, while hadron phenomenology puts the value of the quark mass at low momenta to an effective $\sim 300 \mathrm{MeV}$. This means that the nonstrange quark mass changes by two orders of magnitude when going from low momentum $k<<\Lambda_{\mathrm{QCD}}$ to high momentum $k>>\Lambda_{\mathrm{QCD}}$ (typically $\Lambda_{\mathrm{QCD}} \sim 210 \mathrm{MeV}$ ).

Obviously, a direct comparison between the QCD prediction of the running quark mass and experimental observation is not possible since quarks are confined and propagate only a distance of order a fermi, too small to detect directly. It is a main goal of modern hadron physics to glimpse properties of the confined, colored quarks from colorless hadron properties. We suggest an indirect way of extracting the power-law behaviour of the quark mass function from the experimentally obtainable masses of $\Delta$

\footnotetext{
a e-mail: timvancauteren@gmail.com
}

resonances. Two main ideas are applied in order to do this: insensitivity to chiral symmetry breaking leading to the appearance of parity doublets in the hadron spectrum [13] and linking the mass splittings between parity partners with the running quark mass.

Our proposal can be concisely understood with the help of figure 1 .

\section{Chiral quartets}

Chiral symmetry in the strong interactions is the Noether symmetry related to the chiral transformation, which reads on the classical fermion fields

$$
\psi \rightarrow e^{i \alpha^{a} \tau^{a} \gamma_{5}} \psi
$$

with $\alpha^{a}$ the parameters describing the rotation in $S U(2)$ flavour space with generators $\tau^{a}$. Due to the Dirac matrix $\gamma_{5}$, the chiral transformation is a helicity-dependent rotation in flavour space.

One term in the QCD Lagrangian which is clearly not invariant to the chiral transformation of Eq. 1, is a quark mass term

$$
m_{q} \bar{q} q .
$$

This term breaks chiral symmetry explicitly, and the amount of breaking is larger when the quark mass increases. As mentioned before, the small current quark mass of $5 \mathrm{MeV}$ breaks chiral symmetry only slightly. On top of the explicit breaking of chiral symmetry, the strong interactions also show spontaneous chiral symmetry breaking due to the appearance of the quark condensate $<\bar{q} q>$ (a constituent quark mass of $300 \mathrm{MeV}$ arises and breaks it to a much larger degree). 
EPJ Web of Conferences
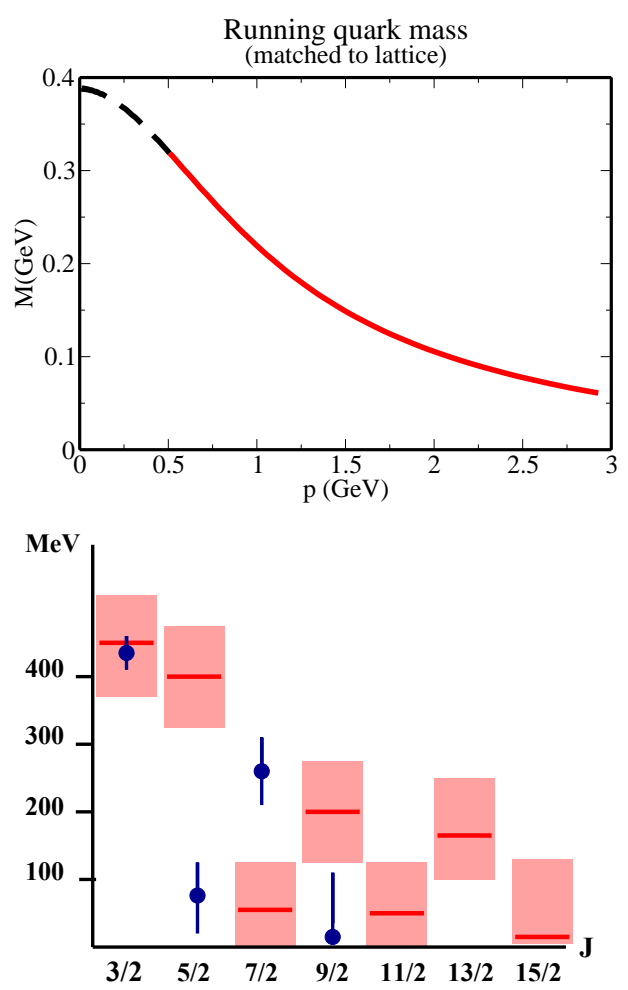

Fig. 1. The running of the quark mass (top), falling with momentum as a power law, is accessible from the hadron spectrum in the region $m(k)<\langle k\rangle$ (continuous red line). All one needs to do is fit a power law to the decreasing parity splittings for excited baryon states (bottom, where we show a variational-Montecarlo model calculation thereof as boxes and experimental data from Ref. [4] as circles). Then the exponent of the quark mass power-law in Eq. (10) is obtained from Eq. (9).

This is the QCD explanation for the absence of parity doubling in the low spectrum, as the symmetry is spontaneously broken. The community now believes that the highly excited states however are insensitive to this spontaneous breaking (see fig. 2 for a classical analogy). Indeed, since the quark mass is large at low momenta and small at high momenta, one may expect that the chiral symmetry breaking is less important in systems where the average quark momentum $<k>$ is high, than in systems where $\langle k\rangle$ is large. This leads to the idea of insensitivity to chiral symmetry breaking high in the hadron spectrum $[1-3,5,6]$ : in highly excited meson or baryon states, the average quark momentum can become larger than $\Lambda_{\mathrm{QCD}}$ and the explicit chiral-symmetry breaking quark mass term is small. This is illustrated in Fig. 3, where typical quark momentum distributions are shown for the lowest-lying $\Delta$ resonance for different spins, together with the lattice QCD calculation of the running quark mass from Ref. [7].

\subsection{Series expansion in $m(k) / k$}

In order to assess the effect of a nonzero quark mass to a hadron mass, it is important to note that the quark mass
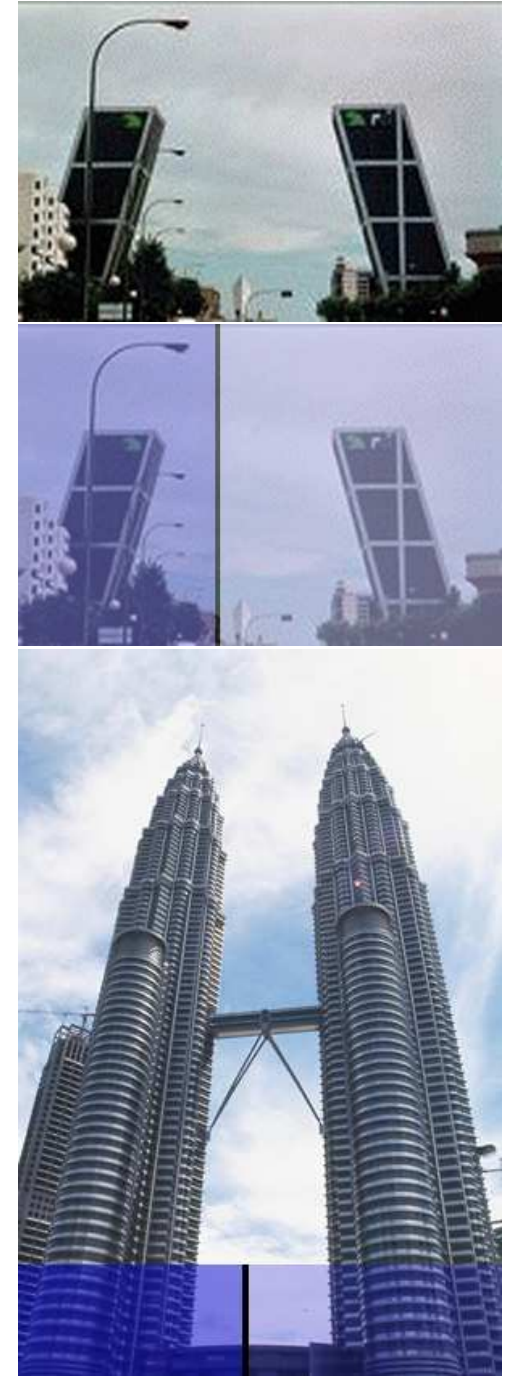

Fig. 2. A classical analogy of three symmetry realizations in a quantum theory. Top: the two buildings have equal mass as required by Reflection symmetry in Wigner mode. Middle: if inmersed in a fluid (non-trivial ground state) with a density gradient from left to right, the two buildings, filled with it, have now different mass (the symmetry is in Goldstone mode). Bottom: even if the fluid breaks the Reflection symmetry, tall enough buildings have close to equal masses (insensitivity to symmetry breaking).

shows up both in the quark spinors and in the QCD Hamiltonian. Since we want to find out what happens at small quark mass and high momentum, a series expansion of the spinors and Hamiltonian in the parameter $m(k) / k$ seems appropriate.

The spinors are expanded as

$$
\begin{aligned}
& U_{k \lambda}=\frac{1}{2 E(k)}\left[\begin{array}{c}
\sqrt{E(k)+m(k)} \chi_{\lambda} \\
\sqrt{E(k)-m(k)} \sigma \cdot \hat{k} \chi_{\lambda}
\end{array}\right] \\
& \stackrel{\vec{k} \rightarrow \infty}{\frac{1}{\sqrt{2}}}\left[\begin{array}{c}
\chi_{\lambda} \\
\sigma \cdot \hat{k} \chi_{\lambda}
\end{array}\right]+\frac{1}{2 \sqrt{2}} \frac{m(k)}{k}\left[\begin{array}{c}
\chi_{\lambda} \\
-\sigma \cdot \hat{k} \chi_{\lambda}
\end{array}\right],
\end{aligned}
$$

with $E(k)=\sqrt{k^{2}+m(k)^{2}}$. We have kept the leading chirally invariant term and the leading chiral-symmetry breaking term which is necessarily of order $m(k) / k$. Note that the 


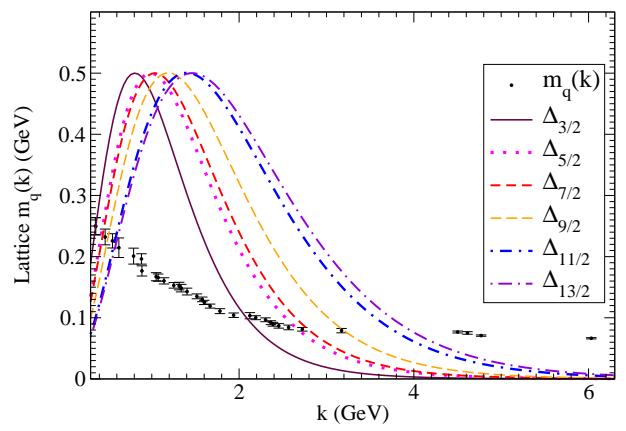

Fig. 3. Typical momentum distributions of increasingly excited $\Delta_{3 / 2}, \cdots, \Delta_{13 / 2}$ resonances overlap less and less with the dynamically generated infrared quark mass. The momentum distributions are computed using variational wave functions (not normalized for visibility) for a linear potential with string tension $\sigma=0.135 \mathrm{GeV}^{2}$.

lower component of the first-order term has the opposite sign of the lower component of the chiral invariant term.

When expanding the QCD Hamiltonian [8] in the weak sense (that is, not of the Hamiltonian itself, but a restriction thereof to the Hilbert space of highly excited resonances, where $\langle k\rangle$ is large), we note that the leading term in the $m(k) / k$ expansion is chiral invariant, while the first order term may involve nonchiral, spin-dependent potentials in the quark-quark interaction:

$$
\left\langle n\left|H^{Q C D}\right| n^{\prime}\right\rangle \simeq\left\langle n\left|H_{\chi}^{Q C D}\right| n^{\prime}\right\rangle+\left\langle n\left|\frac{m(k)}{k} H_{\chi}^{Q C D^{\prime}}\right| n^{\prime}\right\rangle+\ldots
$$

\subsection{Chiral charge and three-quark states}

If the quarks were massless, and there would be no chiral noninvariant mass term in the strong interactions, the chiral charge [9]

$$
Q_{5}^{a}=\int d \mathbf{x} \psi^{\dagger}(\mathbf{x}) \gamma_{5} \frac{\tau^{a}}{2} \psi(\mathbf{x})
$$

would commute with the QCD Hamiltonian. Nevertheless, chiral symmetry would still be spontaneously broken by the ground state, $Q_{5}^{a}|0\rangle \neq 0$, leading to a large quark mass in the quark propagator, pseudo-Goldstone bosons and the loss of parity-degeneracy in ground-state baryons. Using Bogoliubov-rotated quark/antiquark operators $B$ and $D$ and the explicit expression for the spinors, the chiral charge can be written as

$$
\begin{gathered}
Q_{5}^{a}=\int \frac{d^{3} k}{(2 \pi)^{3}} \sum_{\lambda \lambda^{\prime} f f^{\prime} c}\left(\frac{\tau^{a}}{2}\right)_{f f^{\prime}} \frac{k}{\sqrt{k^{2}+m^{2}(k)}} \\
\times\left[(\sigma \cdot \hat{\mathbf{k}})_{\lambda \lambda^{\prime}}\left(B_{k \lambda f c}^{\dagger} B_{k \lambda^{\prime} f^{\prime} c}+D_{-k \lambda^{\prime} f^{\prime} c}^{\dagger} D_{-k \lambda f c}\right)+\right. \\
\left.\frac{m(k)}{k}\left(i \sigma_{2}\right)_{\lambda \lambda^{\prime}}\left(B_{k \lambda f c}^{\dagger} D_{-k \lambda^{\prime} f^{\prime} c}^{\dagger}+B_{k \lambda^{\prime} f^{\prime} c} D_{-k \lambda f c}\right)\right] .
\end{gathered}
$$

The first term between the square brackets represents a quark and antiquark number operators flipping spin and parity. When $m(k)<<k$, it dominates the second term representing the creation or annihilation of a pion (and theRefore realizes chiral symmetry nonlinearly).

As is argumented in Refs. [10-12], repeated action of the chiral charge on a three-quark state leads to a quartet of states, two of each parity, which dynamically breaks into two doublets of parity partners. These partners become degenerate when $m(k)$ vanishes. Moreover, the mass splitting between partners is a direct measure of $m(k)$.

\section{The running quark mass and the $\Delta$ spectrum}

In order to link the mass splitting $\left|M^{P=+}-M^{P=-}\right|$ in a parity doublet to the running quark mass, we will look at the lowest-lying $\Delta$ parity doublets for increasing spin $j$ and we use the following four arguments

1. Regge trajectories: $j=\alpha_{0}+\alpha M^{ \pm 2} \stackrel{j \rightarrow \infty}{\longrightarrow} \alpha M^{ \pm 2}$

2. Relativistic virial theorem [13]: $\left\langle k>\rightarrow c_{2} M^{ \pm} \rightarrow\right.$ $\frac{c_{2}}{\sqrt{\alpha}} \sqrt{j}$

3. The chirally invariant term $\left(<n\left|H_{\chi}^{Q C D}\right| n>\right)$ cancels out in $\triangle M$ :

$$
\left|M^{+}-M^{-}\right| \ll M^{ \pm}
$$

and

$$
\left|M^{+}-M^{-}\right| \rightarrow<\frac{m(k)}{k} H_{\chi}^{Q C D^{\prime}}>\rightarrow c_{3} \frac{m(k)}{k}<H_{\chi}^{Q C D^{\prime}}>
$$

4. In $H_{\chi}^{Q C D}$, the spin-orbit $\mathbf{L}_{i} \cdot \mathbf{S}_{i}$ term is crucial to correct the angular momentum in the centrifugal barrier term from $\mathbf{L}_{i}^{2}$ to the chirally invariant $\mathbf{L}_{i}^{2}+2 \mathbf{L}_{i} \cdot \mathbf{S}_{i}=\mathbf{J}_{i}^{2}-$ $\frac{3}{4}$. Due to the sign difference in the helicity-dependent term $\sim-\sigma \cdot \hat{k}$ in the spinor, the spin-orbit term in $H_{\chi}^{Q C D^{\prime}}$ adds to the mass difference $\Delta M$, instead of cancelling out as it does for $H_{\chi}^{Q C D}$. Since the centrifugal barrier scales like $M^{ \pm}$for high $j$, the spin-orbit term scales with one power of $j$ less:

$$
<H_{\chi}^{Q C D^{\prime}}>\rightarrow c_{5} M^{ \pm} j^{-1} \rightarrow \frac{c_{5}}{\sqrt{\alpha}} \sqrt{\frac{1}{j}} .
$$

Combining these four arguments, we obtain

$$
\left|M^{+}-M^{-}\right| \rightarrow \frac{c_{3} c_{5}}{c_{2} \sqrt{\alpha}} m(<k>) j^{-1} .
$$

An experimental extraction can be done by fitting the exponent $-i$ of $j$ in the splitting

$$
\left|M^{+}-M^{-}\right| \propto j^{-i} \text {. }
$$

Then, the power-law behaviour of the running quark mass is given by

$$
m(k) \propto k^{-2 i+2} .
$$

The experimentally known masses of lowest-lying $\Delta$-resonances for $j=1 / 2, \cdots, 15 / 2$ are shown in Fig. 4. From this, it is clear that the present state of our experimental knowledge of the $\Delta$-spectrum is not sufficient to derive the exponent $i$. Knowledge of the masses of the parity doubler for spins $j>9 / 2$ would greatly enhance this. 


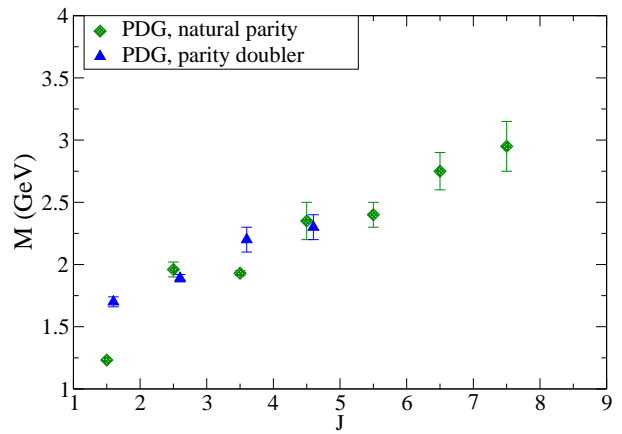

Fig. 4. Experimentally known masses for the lowest-lying $\Delta$ resonance for spins up to $15 / 2$ and each parity. The degeneracy seen at $j=9 / 2$ can be accidental and may be confirmed if the masses of the parity doublers at $j>9 / 2$ are measured.

\section{A basis for $\Delta$ states}

The idea of extracting the running quark mass from the hadron spectrum can be investigated using a chirally invariant quark model. The Hamiltonian we have used in Ref. [10] comes from a field theory upgrade of the Cornell model where we neglect the gluon fields and use a linear quark-quark potential $V_{L}(r)=\sigma r$ with string tension $\sigma=0.135 \mathrm{GeV}^{2}$. This leads to an analytic expression for the mass splitting between parity partners of

$$
\begin{array}{r}
M_{+}-M_{-}=3 \int \frac{d^{3} k_{1}}{(2 \pi)^{3}} \frac{d^{3} k_{2}}{(2 \pi)^{3}}\left(\frac{2}{3}\right) \int \frac{d^{3} q}{(2 \pi)^{3}} \\
\times \hat{V}(q) \frac{1}{2}\left(\frac{m\left(\left|\mathbf{k}_{1}\right|\right)}{\left|\mathbf{k}_{1}\right|}+\frac{m\left(\left|\mathbf{k}_{1}+\mathbf{q}\right|\right)}{\left|\mathbf{k}_{1}+\mathbf{q}\right|}\right) F^{* \lambda_{1} \lambda_{2} \lambda_{3}}\left(\mathbf{k}_{1}, \mathbf{k}_{2}\right) \\
\times\left(\mathbb{I}-\sigma \hat{\mathbf{k}}_{1} \sigma \mathbf{k}_{1}+\mathbf{q}\right)_{\lambda_{1} \mu_{1}} F^{\mu_{1} \lambda_{2} \lambda_{3}}\left(\mathbf{k}_{1}+\mathbf{q}, \mathbf{k}_{2}-\mathbf{q}\right) .
\end{array}
$$

Here $\hat{V}$ is the Fourier transform of the linear potential which falls like $q^{-4}$, and the $F$ is the wavefunction of one of the parity partners. This wavefunction can be obtained variationally as done in Ref. [10]. However, it is only possible to do this for the lowest state for each spin and parity. Higher-lying states can be computed by diagonalizing the Hamiltonian using a set of basis functions for three-quark states.

Constructing an orthonormal basis of three-quark states is not an easy task. The basis functions need to form an antisymmetrized and orthonormal set of states of definite spin and parity. We have built this basis starting from basis states which are a combination of harmonic-oscillator (HO) states with parameter $\alpha$ and a $2 \times 2 \times 2=8$-component spinor

$$
\begin{aligned}
& \mathcal{B}_{N, l, m_{\rho}, m_{\lambda}, R_{i}}^{\alpha}\left(\mathbf{p}_{\rho}, \mathbf{p}_{\lambda}, S_{i}\right) \\
& =\varphi_{n_{\rho}^{N, l}, l_{\rho}^{N, l, m_{\rho}}}^{\alpha}\left(\mathbf{p}_{\rho}\right) \varphi_{n_{\lambda}^{N, l, l}, l_{\lambda}^{N, l}, m_{\lambda}}^{\alpha}\left(\mathbf{p}_{\lambda}\right) \chi_{R_{i}}\left(S_{i}\right),
\end{aligned}
$$

where $\mathbf{p}_{\rho}$ and $\mathbf{p}_{\lambda}$ are the relative Jacobi momenta, and the indices are needed to denote the quantum numbers of the $\mathrm{HO}$ wavefunctions and the spinor.

The following subsequent steps are taken in constructing the basis
- A unitary transformation is applied to the basis functions of Eq. 12, using Clebsch-Gordan coefficients to ensure a fixed spin of the basis function. The parity of the state is automatically fixed for the HO basis functions: HO shells with even (odd) shell number $N$ give rise to positive (negative) parity states.

- The resulting set of basis functions are symmetrised (the colour part of the wavefunction is taken to be antisymmetric and is left out in this discussion) by simply summing over the six possible quark permutations. This gives rise to an overdetermined set of non-orthogonal symmetrized basis functions of fixed spin and parity.

- An orthonormal set of basis functions is created by performing a Gram-Schmidt procedure on the non-orthogonal set. For this, one has to compute the overlaps between the non-orthogonal wavefunctions using the van Beveren-Ribeiro-Moschinsky coefficients [14].

We have performed this orthonormalization procedure and diagonalized the model Hamiltonian for spin $1 / 2$ and $3 / 2$ states of each parity. The resulting masses are plotted in Fig. 5. Here, we can already see a tendency of decreasing mass difference between states of different parity when looking at higher-excited states.
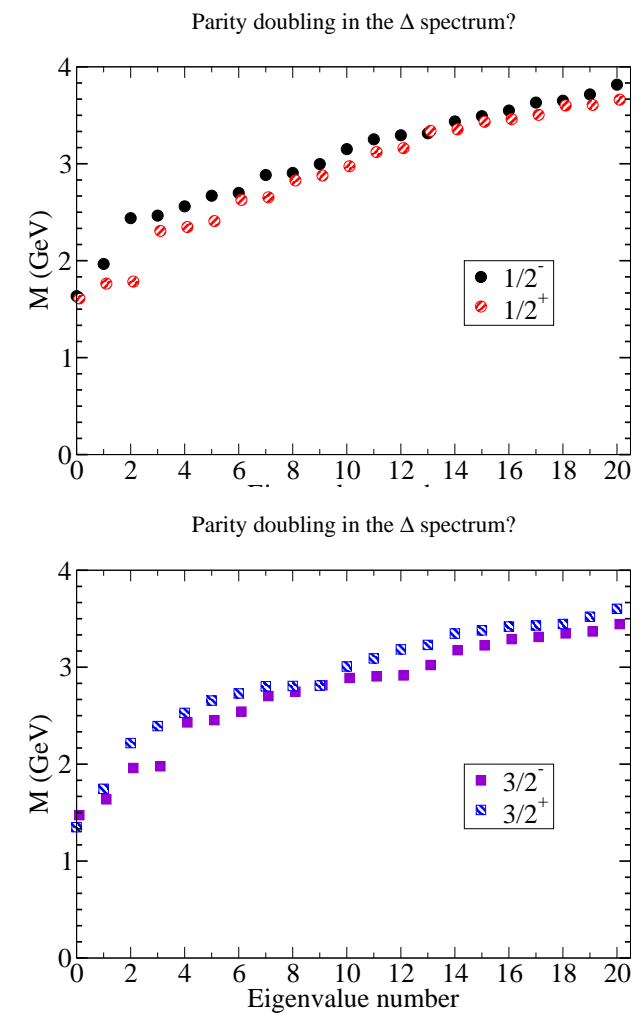

Fig. 5. A massive variational-Montecarlo model calculation [15] allows to check that parity doubling occurs high in the high baryon spectrum, here $\Delta$ baryons with spin $1 / 2$ and $3 / 2$. The computation of highly excited states is currently impossible in lattice gauge theory. Even in our simplified chiral field-theory model of QCD, the present computation is formidable and required $\sim 10^{5}$ CPU-hours at 2-3 GHz. 
$19^{\text {th }}$ International IUPAP Conference on Few-Body Problems in Physics

However, it is at this point not clear which states are parity partners since the density of states is so high. In order to disentangle the pairs in model terms, one just needs to check out the overlaps of the chiral charge between positive and negative parity candidates. If $\left\langle B^{-}\left|Q_{5}^{a}\right| B^{+}\right\rangle \sim 1$, the states are close to being such degenerate partners. However this is difficult to implement with experimental data alone.

Thus we advocate reaching high excitation not by "radial" excitations (successive resonances of increasing mass but equal quantum numbers), but instead by angular excitations (the ground state in each $J$-channel). It is straightforward to match the ground states in an experimental analysis, as long as good partial wave expansions are achieved. We look forward for JLAB and ELSA providing these excited $\Delta_{J}$ spectrum.

\section{Conclusions}

It is commonly quoted in textbooks that symmetries in a quantum theory can be realized in Wigner mode (degenerate spectrum), Goldstone mode (vacuum spontaneously breaks symmetry, Goldstone bosons present) or anomalously (the quantum effective action has less symmetry than the classical action). Chiral symmetry in QCD is widely believed to be in Goldstone mode.

In this note we have argued that actually, in QCD, chiral symmetry is realized in both Goldstone mode (lower part of the spectrum) and Wigner mode (excited states), and given a classical analogy.

We have further commented on the possibility of employing a new perturbative regime in QCD, an expansion in powers of $m(k) / k$ for small quark masses. This is useful for observables that (in Wigner mode) do not receive a contribution from the first order term. Such observables are parity splittings in excited states, their pion couplings, etc. These observables are then able to probe the running quark mass in first order.

We have undertaken a major model calculation of excited baryon states, in the simplest possible model that simultaneously implements chiral symmetry yet has excited states (the first condition rules out non-relativistic quark models, the second the Nambu-Jona-Lasinio model). Thus one needs to resort to global-color models that are nonlocal, and we employ the field-theory upgrade of the Cornell quark model. In this contribution we have shown results for highly excited $\Delta$ baryons to more than 20 eigenvalues (out of reach for conceivable lattice calculations), with a much larger variational basis in the hundred-more range of basis vectors. Our computations, for both spin $1 / 2$ and $3 / 2$, clearly show the parity doubling.

However, this avenue is not promising for experimental extraction, as it is then difficult to match the partners since the density of states is large, and they overlap as their width grows high in the spectrum. We advocate an experimental measurement of the doubling for the ground-states of each angular momentum channel at JLAB and ELSA.

With the data in hand, one can then use our $m(k) / k$ firstorder expansion to obtain the running of the quark mass in the infrared, a hitherto unaccessed quantity in QCD. Other efforts are underway to access this interesting property of a confined object from hadron structure data ${ }^{1}$ and a comparison should prove interesting.

\section{Acknowledgments}

Work supported by grant numbers UCM-BSCH GR58/08 910309, FPA2007-29115-E, FPA2008-00592, FIS200801323, CERN/FP /83582/2008, POCI/FP /81933/2007, /81913/2007, PDCT/FP /63907/2005 and /63923/2005, Spain-Portugal bilateral grant HP2006-0018 / E-56/07, as well as the Flanders Research Foundation (FWO). FLE acknowledges useful conversations with Craig Roberts, Eric Swanson and Christoph Hanhart during the recent BadHonnef meetings.

\section{References}

1. L. Y. Glozman, Phys. Lett. B 475 (2000) 329.

2. E. S. Swanson, Phys. Lett. B 582, (2004) 167.

3. R. F. Wagenbrunn and L. Y. Glozman, Phys. Lett. B 643, (2006) 98; T. D. Cohen and L. Y. Glozman, Mod. Phys. Lett. A 21, (2006) 1939; L. Y. Glozman, A. V. Nefediev and J. E. F. Ribeiro, Phys. Rev. D 72, (2005) 094002.

4. C. Amsler et al. [PDG], Phys. Lett. B 667, (2008) 1.

5. A. Le Yaouanc et al., Phys. Rev. D 31 (1985) 137.

6. P. Bicudo et al., Phys. Lett. B 442, (1998) 349.

7. P.O. Bowman et al., Nucl. Phys. B, Proc. Suppl. 161, (2006), 27; M.B. Parappilly et al., Phys. Rev. D73, (2006) 054504; S. Furui, Few-Body Syst. 45, (2009) $51 ; \mathbf{4 6},(2009) 73$.

8. For a discussion on $H^{\mathrm{QCD}}$, a good starting point is $\mathrm{N}$. H. Christ and T. D. Lee, Phys. Rev. D 22, (1980) 939.

9. A. V. Nefediev, J. E. F. Ribeiro and A. P. Szczepaniak, JETP Lett. 87, (2008) 271.

10. P. Bicudo, M. Cardoso T. Van Cauteren and Felipe J. Llanes Estrada, Phys. Rev. Lett. 103, (2009) 092003.

11. C. E. DeTar and T. Kunihiro, Phys. Rev. D 39, (1989) 2805; D. Jido, T. Hatsuda and T. Kunihiro, Phys. Rev. Lett. 84, (2000) 3252.

12. T. D. Cohen and L. Y. Glozman, Phys. Rev. D 65, (2001) 016006.

13. W. Lucha and F. F. Schoberl, Mod. Phys. Lett. A 5, (1990) 2473.

14. E. van Beveren, Z. Phys. C 17, (1983) 135.

15. T. Hahn, Comput. Phys. Commun. 168, (2005) 78.

\footnotetext{
${ }^{1}$ Craig Roberts, private communication
} 